\title{
Molecular Dynamics Simulation on Structure and Dielectric Permittivity of $\mathrm{BaTiO}_{3} /$ PVDF Composites
}

\author{
Ruitian Bo, ${ }^{1}$ Junwang Liu, ${ }^{1}$ Chunfeng Wang, ${ }^{1}$ Yongliang Wang, ${ }^{1}$ Peigang He, \\ and Zhidong Han $\mathbb{1}^{1,3}$ \\ ${ }^{1}$ School of Materials Science and Engineering, Harbin University of Science and Technology, China \\ ${ }^{2}$ School of Materials Science and Engineering, Harbin Institute of Technology, China \\ ${ }^{3}$ Key Laboratory of Engineering Dielectrics and Its Application, Ministry of Education, Harbin University of Science and Technology, \\ 150080, China
}

Correspondence should be addressed to Zhidong Han; zhidong.han@hrbust.edu.cn

Received 21 July 2021; Accepted 12 September 2021; Published 22 September 2021

Academic Editor: Sébastien Déon

Copyright (c) 2021 Ruitian Bo et al. This is an open access article distributed under the Creative Commons Attribution License, which permits unrestricted use, distribution, and reproduction in any medium, provided the original work is properly cited.

Molecular dynamics (MD) simulation was performed to investigate the structure and dielectric permittivity of poly(vinylidene fluoride)- (PVDF-) based composites with different contents of barium titanate (BT). The $\beta$-phase PVDF model with 100 structural units and the spherical BT particle model with a radius of $0.495 \mathrm{~nm}$ were built and applied in the initial models with three PVDF macromolecular chains and BT particles for the MD simulations of the BT/PVDF composites. The influences of BT content on the morphological structure, the free volume fraction, and glass transition temperature of the composites were explored according to the simulated results and the experimental ones of X-ray diffraction (XRD) and scanning electron microscope (SEM). A model was proposed to predict the static dielectric permittivity of the composites, the results of which were compared with the Cole-Cole fitting results of dielectric spectroscopy. Attempts were made to reveal the structure evolution and the micropolarization mechanism with the increasing content of BT.

\section{Introduction}

Polymer-based dielectric composites have been widely applied in the industries of information, energy, electrical, and electronics thanks to their good processability, excellent mechanical properties, low dielectric loss, and high breakdown strength $[1,2]$. Recently, polymer-based composites with high dielectric permittivity have attracted great attention [3-7]. To obtain the composites, adding ceramic particles with high dielectric permittivity into the polymeric matrices is considered to be one of the most common and promising strategies, which takes the advantages of colossal permittivity of ceramic particles and good dielectric strength of polymers [8]. Poly(vinylidene fluoride) (PVDF), a ferroelectric polymer with relatively high dielectric permittivity $[9,10]$, was widely investigated in the formation of the dielectric composites with addition of ferroelectric ceramic fillers, such as $\mathrm{BaTiO}_{3}, \mathrm{SrTiO}_{3}$, and $\mathrm{Ba}_{0.6} \mathrm{Sr}_{0.4} \mathrm{TiO}_{3}$ [11-18].
A dielectric permittivity of 27.9 at $1 \mathrm{kHz}$ and breakdown strength of $117 \mathrm{kV} / \mathrm{mm}$ were reported by $\mathrm{Ma}$ et al. [19] for PVDF-based composites with $30 \mathrm{vol} \%$ core-shell structured fillers of vinyl functionalized $\mathrm{BaTiO}_{3}$ wrapped with thiolterminated PVDF. Wang et al. [20] reported a high dielectric permittivity of 97.4 at $100 \mathrm{~Hz}$ in PVDF-based nanocomposites with $60 \mathrm{vol} \% \mathrm{Ba}\left(\mathrm{Fe}_{0.5} \mathrm{Ta}_{0.5}\right) \mathrm{O}_{3}$. Hu et al. [21] prepared PVDF composites with hydroxylated $\mathrm{Ba}_{0.6} \mathrm{Sr}_{0.4} \mathrm{TiO}_{3}$ (BST) as filler and reported the dielectric permittivity of 40 at $1 \mathrm{kHz}$ and the loss tangent of 0.19 for the composites with $40 \mathrm{vol} \%$ BST.

To date, remarkable progresses have been achieved in the investigation of polymer-based composites with high dielectric permittivity. Meanwhile, some studies on the polarization mechanism and the simulations on dielectric permittivity were attempted [22, 23]. Molecular dynamics (MD) simulation on the basis of classical mechanics equations and quantum calculations is capable of investigating 
the motion of the atoms in the simulation system and shows great potential in studying dielectric permittivity [24-27]. Shen et al. [28] established a composite model of epoxy resin (EP) with graphene nanosheets and investigated the dielectric permittivity through MD simulation. Huang et al. [29] calculated the dielectric permittivity of polydimethylsiloxane (PDMS) composites with graphene oxide (GO) by MD simulation. Feng et al. [30] used MD simulations to study the dielectric behaviors of polymer composites with threedimensional particle networks and revealed the increased dielectric permittivity of the composites when the particle network formed and the adjacent particles were polarized due to the continuous coupling effect. Thus, it can be seen that MD simulations would be useful in understanding the polarization mechanism and predicting the dielectric permittivity of the composites, which makes it possible to provide guidance for the design of polymer composites with high dielectric permittivity without the time-consuming and laborious experiments.

In view of the fact that $\mathrm{BaTiO}_{3} / \mathrm{PVDF}$ dielectric composites (BT/PVDF) have been extensively researched while the $\mathrm{MD}$ simulations on the structure and polarization mechanism of the composites are very limited, this paper focuses on the simulation of the structure of BT/PVDF composites and the prediction of dielectric permittivity by MD simulations. Therefore, the simulation model of BT/PVDF composites was built according to the crystalline structure of PVDF, BT, and their composites. The microstructure of the composites with different BT contents was simulated to study the effect of BT content on the microstructure of the composites. The free volume fraction and the glass transition temperature of BT/PVDF composites were calculated by molecular dynamics simulation to reveal the segment motion of macromolecules in the composites. The calculation method of static dielectric permittivity of the composites was established. In comparison with the experimental data, the polarization mechanism of the composites was proposed.

\section{Experimentals}

2.1. Raw Materials. Poly(vinylidene fluoride) (PVDF) was purchased from American 3M Co. Ltd. Barium titanate (BT) with a particle size of $100 \mathrm{~nm}$ was purchased from Aladdin Co. Ltd.

2.2. Preparation of the Composites. BT/PVDF composites were prepared by a melt blending method according to the compositions in Table 1. PVDF was added into a torque rheometer (ZJL-200, Changchun Intelligent Instrument Equipment Co., Ltd.), preheated to $190^{\circ} \mathrm{C}$ at a rotating rate of $60 \mathrm{rpm}$, and blended for 5 minutes to prepare PVDF sample. PVDF and BT particles were added to the torque rheometer, preheated to $190^{\circ} \mathrm{C}$, and mixed at $60 \mathrm{rpm}$ for 20 minutes to obtain BT/PVDF composites. The PVDF and composites were melt pressed with a flat vulcanizer (XLB25-D, Huzhou Shuangli Automation Technology Equipment Co., Ltd.) at $190^{\circ} \mathrm{C}$ and $15 \mathrm{MPa}$ for 15 minutes and naturally cooled to
TABle 1: Compositions of BT/PVDF composites.

\begin{tabular}{lcc}
\hline Sample & PVDF (vol\%) & BT (vol\%) \\
\hline BT10/PVDF & 90 & 10 \\
BT15/PVDF & 85 & 15 \\
BT20/PVDF & 80 & 20 \\
\hline
\end{tabular}

room temperature under a pressure of $15 \mathrm{MPa}$ to obtain the samples for further characterization.

2.3. Characterization. A field emission scanning electron microscope (SEM, Sirion200, FEI) was used to observe the morphological structure of the composites with the sample fractured in liquid nitrogen and the section metalized with gold. X-ray diffractometer (XRD, D/max- $\gamma \mathrm{B}$, Rigaku Electric) was used to characterize the crystalline structure of the composite at a voltage of $40 \mathrm{kV}$ and a current of $30 \mathrm{~mA}$ with the step of $0.013^{\circ}$ and the scanning speed of $8^{\circ} / \mathrm{min}$ in the $2 \theta$ range of $5^{\circ}-90^{\circ}$. A broadband dielectric spectrometer (Alpha-A, Novocontrol, Germany) was used to analyze the dielectric permittivity and loss at room temperature at a voltage of $3 \mathrm{~V}$ in the frequency range of $1 \sim 10^{7} \mathrm{~Hz}$ on the sample coated with circular aluminum electrodes with a diameter of $25 \mathrm{~mm}$ by a high-vacuum resistance evaporation coating machine (Model ZHD-400, Beijing Techno Technology Co., Ltd.).

\section{Molecular Dynamics Simulation}

3.1. Crystalline Structure. PVDF, a semicrystalline polymer, has a variety of crystalline forms, among which the $\beta$-phase structure is desirable for dielectric composites due to its ferroelectric characteristic. The crystalline structure of PVDF shows dependence on synthesis methods, melting temperature, processing conditions, and annealing conditions [31]. PVDF and its composites were prepared by the melt blending method, and the XRD patterns of the composites are shown in Figure 1. As a result, PVDF prepared by the melt blending method has a polycrystalline structure. The peaks at $2 \theta$ of $17.8^{\circ}, 18.4^{\circ}, 19.9^{\circ}$, and $26.6^{\circ}$ are assigned to (100), (020), (110), and (021) diffractions of $\alpha$-phase PVDF [32]. The peak at $2 \theta$ of $36.1^{\circ}$ is assigned to (001) diffraction of $\beta$-phase and that at $2 \theta$ of $38.7^{\circ}$ to $(020)$ diffraction of $\gamma$ phase. BT (PDF No. 31-174) has a typical cubic crystalline structure and shows the diffraction peaks at $2 \theta$ of $22.1^{\circ}$, $31.5^{\circ}, 38.8^{\circ}, 45.2^{\circ}, 50.8^{\circ}$, and $56.2^{\circ}$, corresponding to (100), (110), (111), (200), (210), and (211) diffractions, respectively. For BT/PVDF composites with different contents of $\mathrm{BT}$, the strong diffraction peaks of BT are observed while the diffraction peaks of PVDF are relatively weak. A diffraction peak at $2 \theta$ of $20.2^{\circ}$ assigned to (110) diffraction of $\beta$ phase PVDF [33] is observed and strengthened with the increasing content of $\mathrm{BT}$, which indicates the preferential growth of $\beta$-phase crystalline structure in the composites. No emergence of new diffraction peaks suggests the physical interaction between BT and PVDF. 


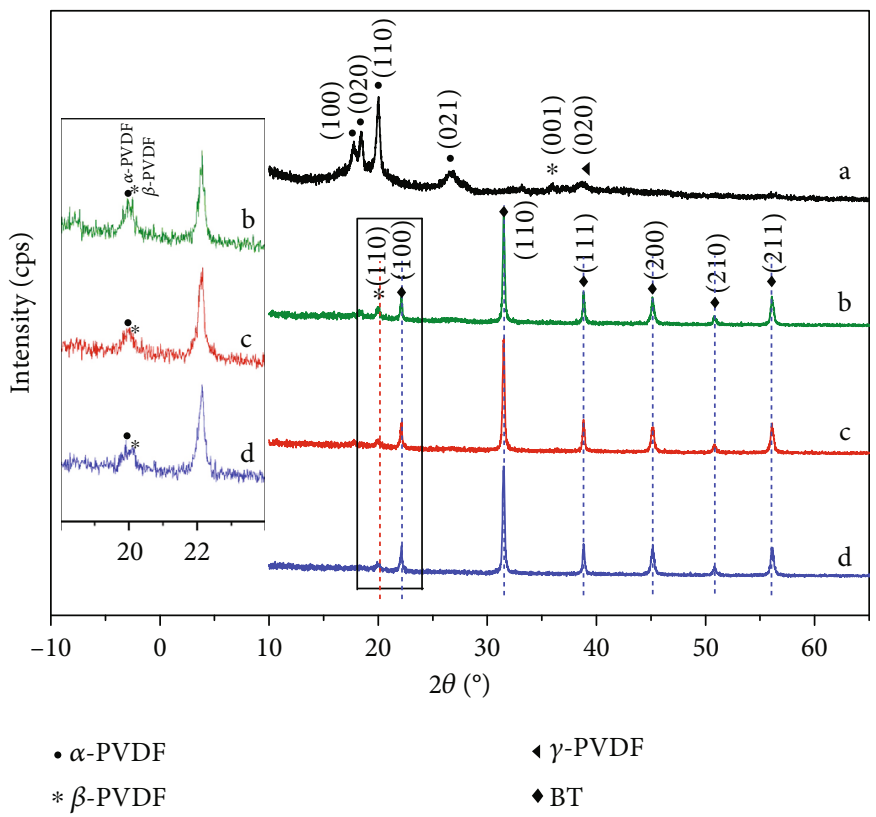

Figure 1: XRD patterns of PVDF (a), BT10/PVDF (b), BT15/PVDF (c), and BT20/PVDF (d).

3.2. Model Building. According to the XRD results in Figure 1, the polycrystalline structure of PVDF and the cubic crystalline structure of BT could be inferred in the BT/PVDF composites. Considering that $\beta$-phase crystalline structure of PVDF has the highest degree of polarization among all the crystalline forms, a macromolecular chain model of PVDF was constructed according to the $\beta$-phase crystalline structure (Figure 2(a)), which was composed of 100 structural units. The $\beta$-phase PVDF molecular chain conformation adopts the all-trans- (TTT) serrated conformation, as shown in Figure 2(b). Meanwhile, a cubic BT structure with a space group of $\mathrm{Pm} 3 \mathrm{~m}$ was used to establish a spherical BT particle model with a radius of $0.495 \mathrm{~nm}$. The ball-and-stick model and scale model of BT spherical particles are shown in Figure 2(c). Three PVDF macromolecular chains and $\mathrm{BT}$ particles were used to construct the models of BT/PVDF composites. Figure 2 shows the initial models of PVDF, BT, and BT20/PVDF.

The rationality of the initial models was evaluated by the density simulations. Figure 3 shows the density of PVDF and BT20/PVDF by MD simulations according to the initial models. As shown in Figure 3(a), the density of PVDF according to the initial model composed of 3 strips of 100 structural units is $1.68 \mathrm{~g} / \mathrm{cm}^{3}$, which is close to the measured density of PVDF $\left(1.71 \mathrm{~g} / \mathrm{cm}^{3}\right)$. As shown in Figure 3(b), the density of BT20/PVDF according to the initial model is $2.00 \mathrm{~g} / \mathrm{cm}^{3}$, which is close to the measured density of BT20/PVDF $\left(2.38 \mathrm{~g} / \mathrm{cm}^{3}\right)$. The established initial models of PVDF and BT20/PVDF are proved to be reasonable and practical while taking into account the complexity of the model and the length of calculation.

3.3. Simulation Details. Material Studio 2019 software was applied to carry out MD simulation by using the Forcite module. After constructing the initial models, the geometric structure was optimized by the Smart algorithm, which combined the steepest descent, conjugate gradient, and Newton minimization algorithm, and the convergence level was set to $0.0001 \mathrm{kcal} / \mathrm{mol}$ in a cascade manner. After the geometric structure was optimized, $\mathrm{MD}$ simulation was performed on the optimized model. Firstly, the model system was annealed and 5 annealing cycles were set; i.e., the system was heated to $500 \mathrm{~K}$ and then cooled back to $298 \mathrm{~K}$ at $40 \mathrm{~K}$ intervals in one cycle. The annealing cycle was performed in the NVT ensemble (constant particle number, constant volume, and constant temperature) with a total of 1000 ps MD simulation. The complete atomic trajectory output results were recorded every $20 \mathrm{ps}$ during the annealing cycle. After annealing simulation, the BT/PVDF model was relaxed to reach the global minimum, and thus, the unreasonable conformations and low-probability conformations were eliminated. Secondly, $2000 \mathrm{ps}$ MD simulation was performed under the condition of $298 \mathrm{~K}$ in the NVT ensemble to balance the system with Nose as the temperature control method. After that, under the conditions of $298 \mathrm{~K}$ and 1 standard atmosphere, $500 \mathrm{ps}$ MD simulation was performed in the NPT ensemble with Nose as the temperature control method, Berendsen as the pressure control method, and Universal Force Field (UFF) as the force field. van der Waals was calculated by an atom-based method. Electrostatic was calculated by a group-based method, and the cut-off radius was $12.5 \AA$. The velocity Verlet algorithm was selected to solve the classical motion equation with a time step of $1 \mathrm{fs}$. Consequently, the NPT dynamic trajectory of 500 ps was used for calculation and structural analysis.

Generally, temperature fluctuations and energy fluctuations were used as the standard to judge whether molecular dynamics simulation has reached equilibrium. If the fluctuation of temperature and energy is within $5 \%$, the system was considered to be in equilibrium. In order to obtain accurate 
(b)

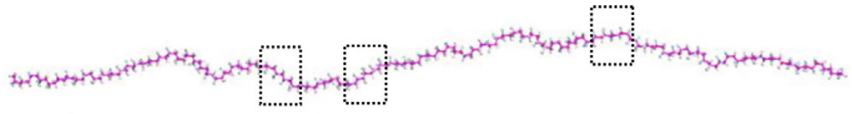

(a)

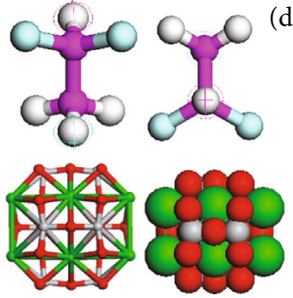

(d)

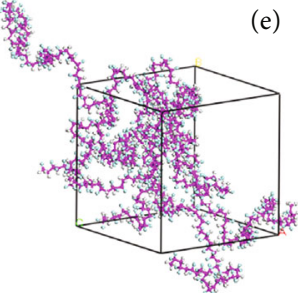

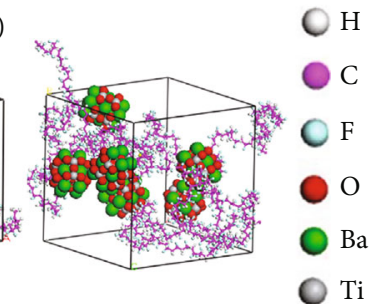

Figure 2: The initial models of PVDF $\beta$-phase structural unit (a), PVDF macromolecular chain (b), BT spherical particle (c), PVDF (d), and BT20/PVDF (e).

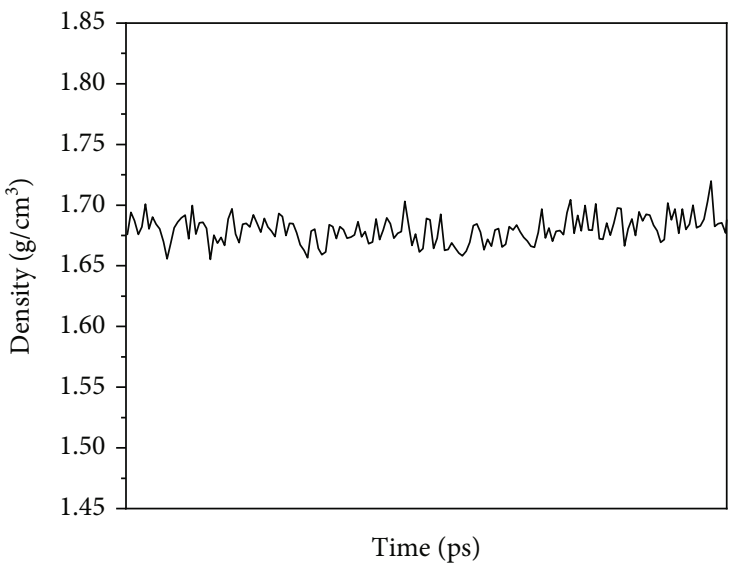

(a)

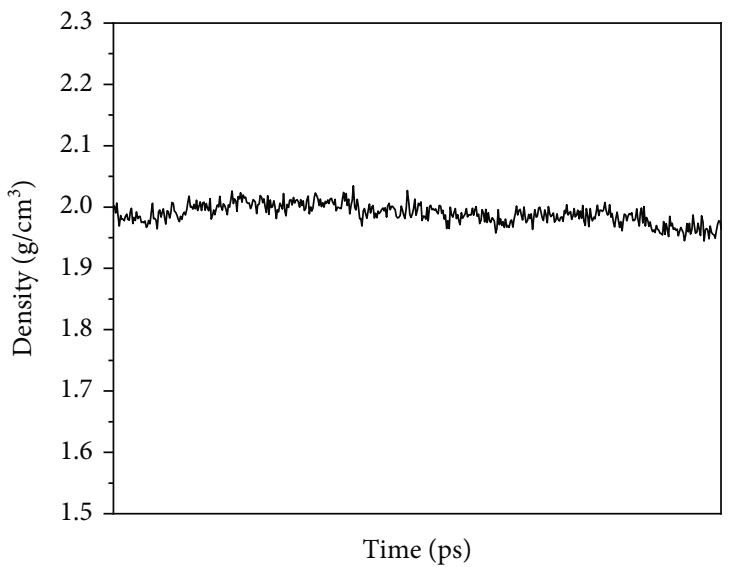

(b)

Figure 3: MD simulation of the final 500 ps PVDF (a) and BT20/PVDF (b) density based on the initial model.

analysis results, all molecular dynamics simulation results in this study were obtained under system equilibrium conditions. Figure 4 shows the energy and temperature curves of the final NPT-MD simulation process taking the PVDF and BT20/PVDF hybrid system as an example. Supplementary figure 1 shows the temperature and energy distribution during the final NPT-MD simulation of BT10/PVDF and BT15/PVDF composites.

Accurate simulation close to the real PVDF model is essential. To verify the balanced PVDF model, we must first obtain specific properties and simulated values through comparative experiments. As shown in Table 2, the cohesive energy density and solubility parameters of PVDF are $4.991 \times 10^{8} \mathrm{~J} / \mathrm{m}^{3}$ and $22.342\left(\mathrm{~J} / \mathrm{cm}^{3}\right)^{0.5}$, respectively. The difference between these data and the actual value from the experiment is small, indicating that the force field and model method used here can reproduce the condensed phase properties of PVDF.

\section{Results and Discussion}

\subsection{Morphological Structure}

4.1.1. Simulated Structure. The simulated structure of the $\mathrm{BT} / \mathrm{PVDF}$ composites with the different BT contents was obtained by MD simulations on the basis of the initial models. Figure 5 compares the initial models and the simulated structure of the composites. For BT10/PVDF, BT particles are well dispersed in the system and PVDF macromolecular chains tend to assemble together. With the increasing content of BT, the agglomeration of BT particles and the assembling of PVDF macromolecular chains are found in the simulated structure of BT15/PVDF. Such phenomena are more remarkable for BT20/PVDF. The initial model of BT20/PVDF describes a uniformly dispersed structure of BT particles and an extended macromolecular chain of PVDF, while the structure after the simulation presents agglomerated BT particles and assembled macromolecular chains. The results indicate the strong influences of BT content on the structure of the composites. The composites with higher BT content than 10 vol\% show the agglomerating tendency of BT particles and the enhanced assembling of PVDF macromolecular chains, which is consistent with the XRD results in Figure 1.

4.1.2. Morphological Analysis. Morphological structure of BT/PVDF composites with different BT contents was investigated by SEM, the results of which are shown in Figure 6. For BT10/PVDF, it can be observed in Figure 6(a) that BT particles are well dispersed in PVDF. The obvious interface 


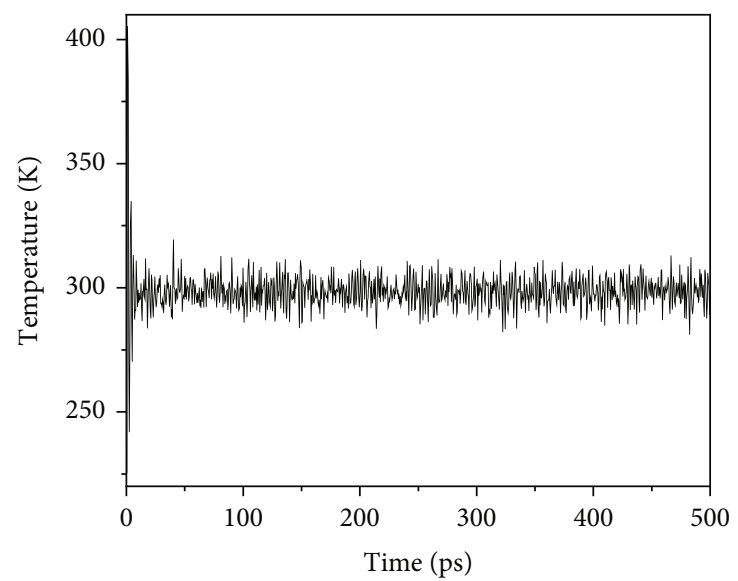

(a)

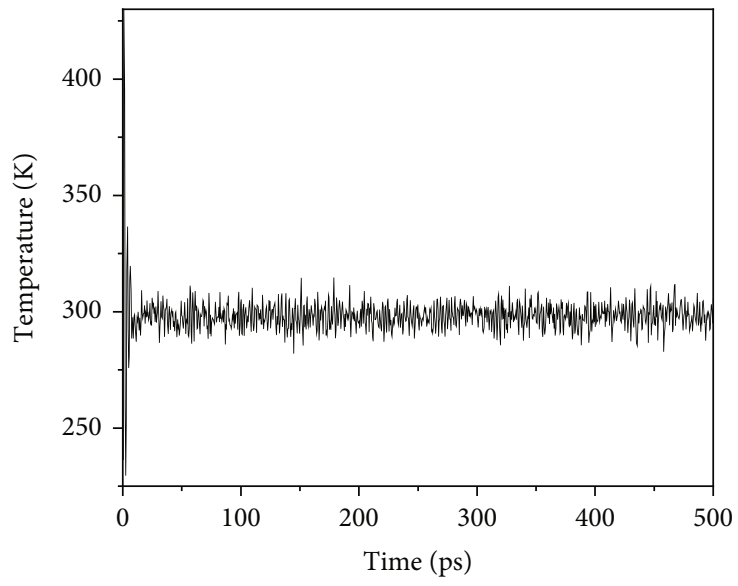

(c)

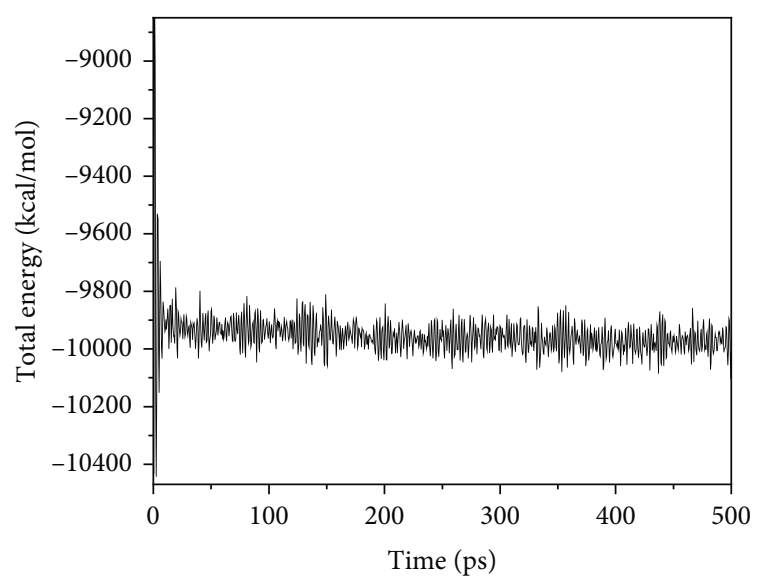

(b)

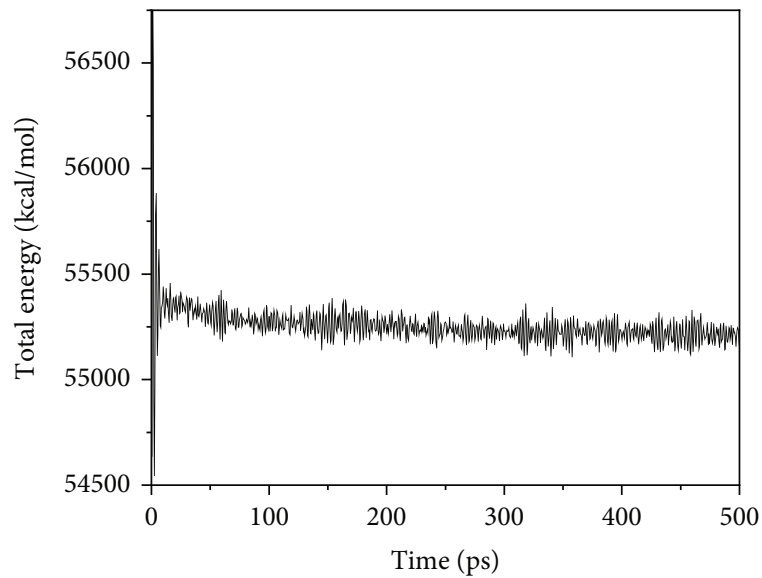

(d)

Figure 4: Temperature and energy distribution during the final NPT-MD simulation of the PVDF (a, b) and BT20/PVDF (c, d) hybrid system.

TABLE 2: Simulated and experimental values of PVDF cohesive energy density and solubility parameters.

\begin{tabular}{lcc}
\hline & $\mathrm{CED} \times 10^{8}\left(\mathrm{~J} / \mathrm{m}^{3}\right)$ & $\sigma\left(\left(\mathrm{J} / \mathrm{cm}^{3}\right)^{0.5}\right)$ \\
\hline Analog value & 4.991 & 22.342 \\
Experimental value [34] & 5.382 & 23.200 \\
\hline
\end{tabular}

structure between BT and PVDF reveals their weak interaction, which can be further evidenced by the holes left due to the abscission of BT particles during the brittle fracture in liquid nitrogen. With the increase of BT content, the agglomeration of BT particles becomes notable for BT15/PVDF as shown in Figure 6(b). The undulating cross section is observed with the fractured cracks formed due to the agglomeration of BT particles and its weak interaction with PVDF. As the BT content increases to $20 \mathrm{vol} \%$, the agglomerated BT particles are full of the fractured section and the matrix of PVDF is hardly observed in the SEM micrographs of BT20/PVDF as shown in Figures 6(c).

Based on the MD simulations and the SEM observations of BT/PVDF composites with different BT contents, it can be inferred that the morphological structure is closely related to the BT content. Both methods come to the common conclusion that the agglomeration of BT particles forms when the BT content is higher than $10 \mathrm{vol} \%$. Furthermore, the MD simulations also reveal the assembling of PVDF macromolecular chains, which gives the reasonable evidence for the XRD results. It follows that MD simulation can be helpful in understanding the change of morphological structure by revealing the assembling process of PVDF macromolecular chains and the agglomeration of BT particle. The simulated structure gives a useful supplement to SEM and XRD results.

\subsection{Fractional Free Volume and Glass Transition Temperature}

4.2.1. Fractional Free Volume. The conformation of PVDF macromolecular chain and its interaction with BT particles are important factors that influence the performances of BT/PVDF composites. Considering the weak interaction between PVDF and BT particles, as evidenced by Figure 6, the spatial structure of the composites including the free volume $\left(V_{\text {free }}\right)$ and the occupied volume $\left(V_{\text {occupied }}\right)$ would be important to understand the segment motion and the 


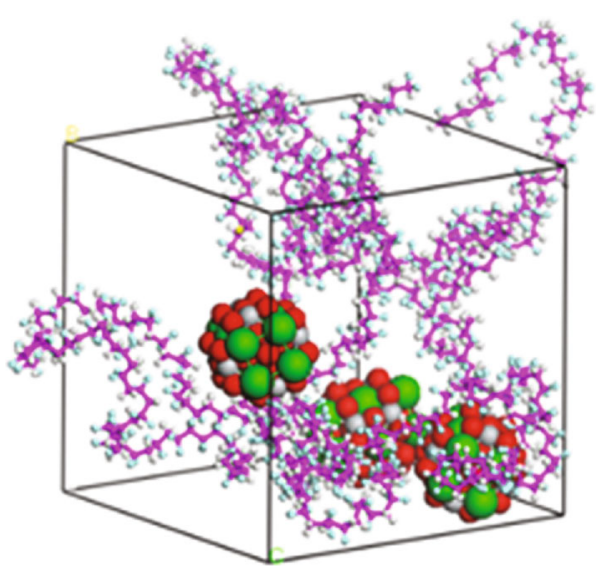

(a)

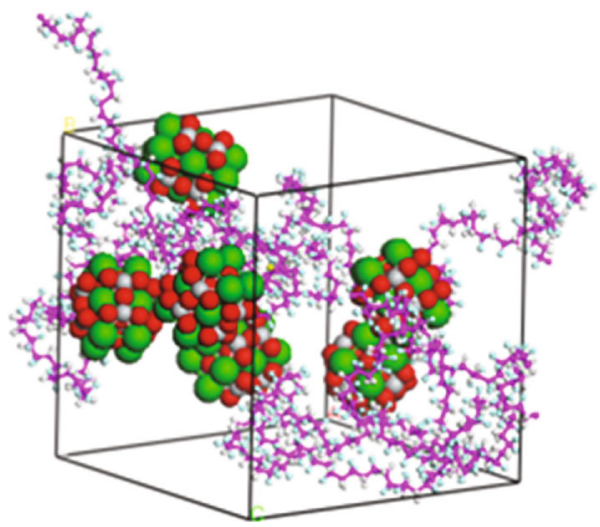

(c)

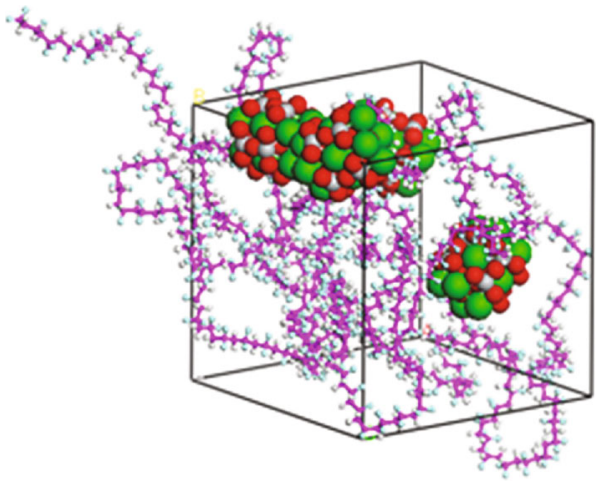

(e)

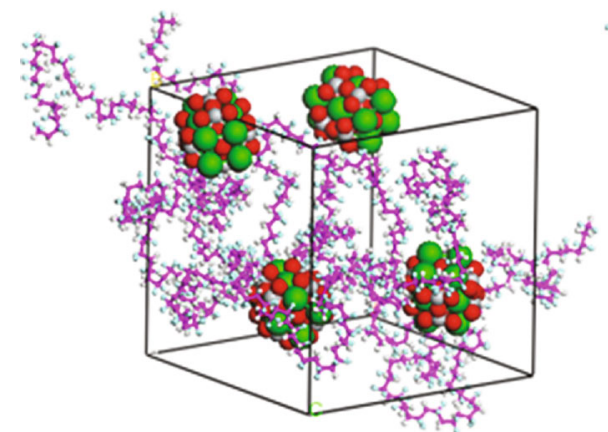

(b)

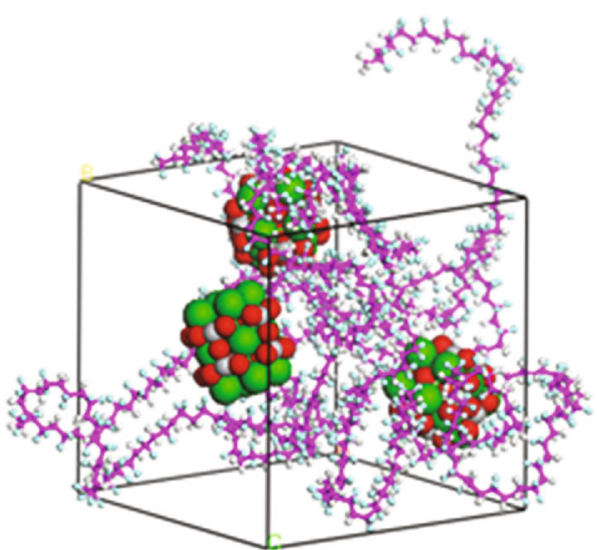

(d)

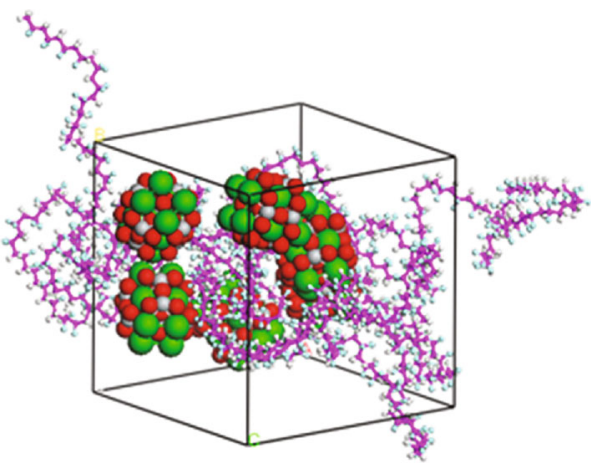

(f)

Figure 5: The initial model $(\mathrm{a}-\mathrm{c})$ and the simulated structure $(\mathrm{d}-\mathrm{f})$ of BT/PVDF composites BT10/PVDF (a, d); BT15/PVDF (b, e); BT20/PVDF (c, f).

conformation of PVDF macromolecular chain. According to the principle shown in Figure 7, the hard ball probe method is used to calculate the free volume of PVDF and BT/PVDF composites. A probe with a radius of $1 \AA$ moves on the van der Waals surface to produce a Connolly surface. The part of the space isolated on the Connolly surface that does not contain the atoms of the system is the free volume.

Figure 8 shows the spatial structure of PVDF and BT/PVDF composites, where the blue area represents the free volume, and the gray area represents the occupied vol- ume. It can be observed that the free volume of the composites with different BT contents shows noticeable difference in terms of its position, shape, and size. Compared with PVDF, the free volume of BT10/PVDF becomes smaller. As the BT content increases, the free volume of BT15/PVDF and BT20/PVDF increases in size when compared with BT10/PVDF. Such phenomenon is more significant for BT20/PVDF. Based on the simulated results in Figure 8, the fractional free volume (FFV) was calculated according to Equation (1) to reveal the effects of the BT content on 


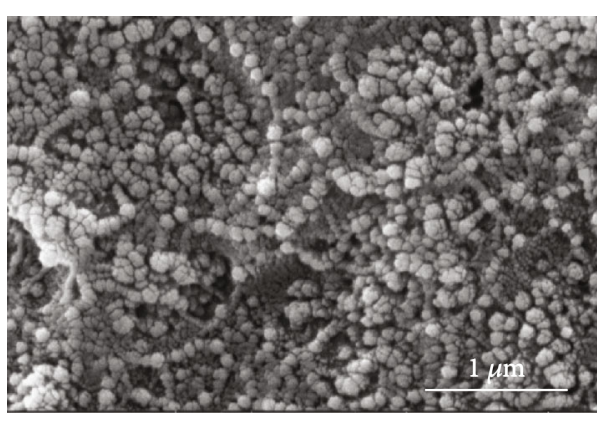

(a)

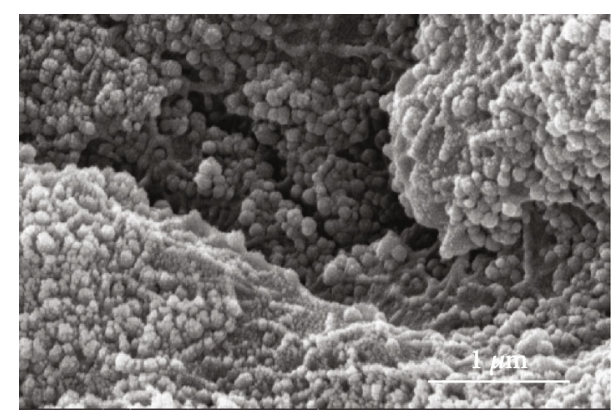

(b)

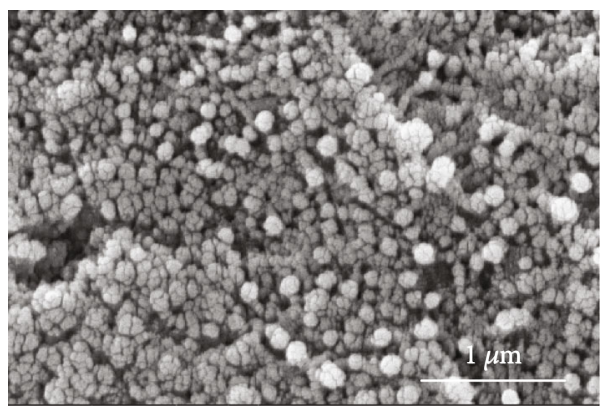

(c)

FiguRE 6: SEM micrographs of the BT/PVDF composites: (a) BT10/PVDF; (b) BT15/PVDF; (c) BT20/PVDF.

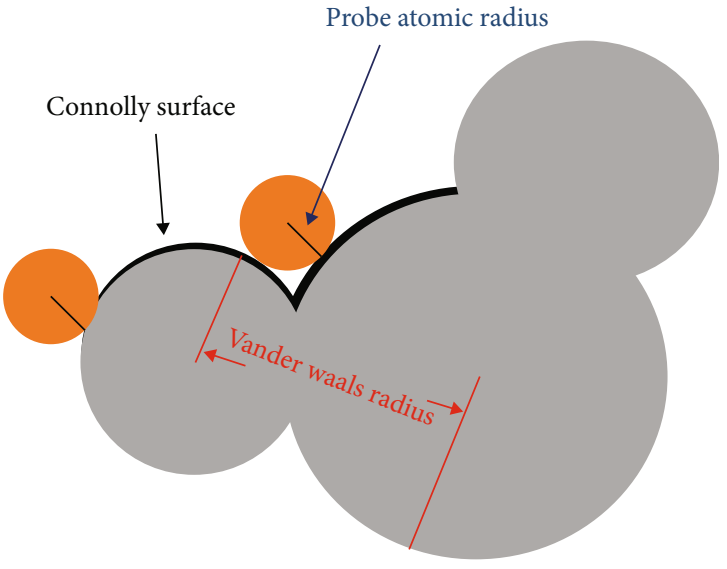

Figure 7: Schematic diagram of Connolly surface.

the conformation of the macromolecular chains [35]. The results are shown in Table 3.

$$
\mathrm{FFV}=\frac{V_{\text {free }}}{V_{\text {total }}}
$$

where $V_{\text {total }}$ is the total volume of the model and $V_{\text {free }}$ is the free volume of the model.

According to the FFV values in Table 3, it can be found that the FFV of PVDF with the value of $28.22 \%$ is higher than that of BT10/PVDF. As the BT content increases from $10 \mathrm{vol} \%$ to $20 \mathrm{vol} \%$, the FFV value increases from $20.65 \%$ of BT10/PVDF to $24.95 \%$ of BT20/PVDF. However, the FFV of BT20/PVDF is still lower than that of PVDF. As observed in Figure 8, the gathering of BT particles and PVDF macromolecular chains can be found in the spatial structure of the composites, which indicates that the BT particles occupy a considerable part of the volume and result in the decreased free volume. With the increasing BT content, the agglomeration of BT particles, together with the assembling of PVDF macromolecular chains as shown in Figure 5, leads to the release of the free volume. As a result, the FFV of BT20/PVDF increases comparatively.

Therefore, the MD simulation is capable of mimicking the spatial structure of the BT/PVDF composites and the calculated results reflect the effects of BT particles on the FFV, which could be related to the segment motion of macromolecular chain. The free volume is the intermolecular space among the materials, which provides space to enable the movement of the macromolecular chain. The introduction of BT particles reduced the FFV of the composites, which hindered the segment motion and made it difficult to change the conformation of the macromolecular chains. Thus, the performance of the composites related to the segment motion and the conformation of the macromolecular chain would be changed, such as glass transition temperature $\left(T_{\mathrm{g}}\right)$.

4.2.2. Glass Transition Temperature. $T_{\mathrm{g}}$ is a property of the amorphous materials or the amorphous portion of a semicrystalline materials, which is described as the temperature at which the amorphous regions experience transition from a rigid state to a more flexible state. $T_{\mathrm{g}}$ of PVDF and BT/PVDF composites was studied by MD simulation. The specific method was on the basis of the simulation details in Section 3.3. After the simulation of 2000 ps in the NVT ensemble, the following processes were performed. Firstly, the initial temperature was set to $550 \mathrm{~K}$, and then, the system 


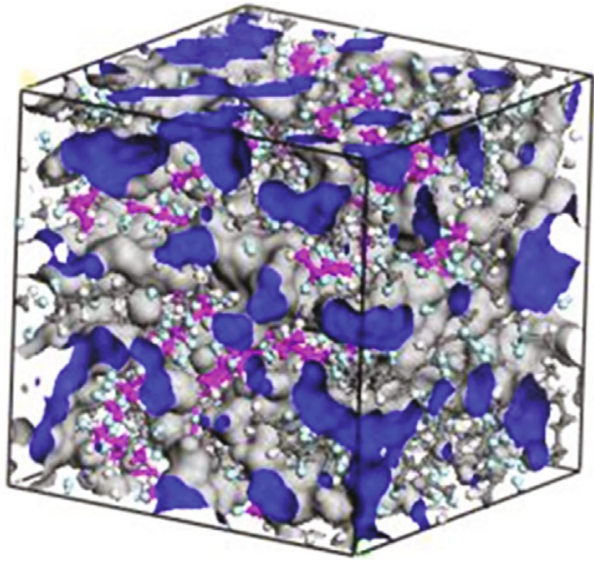

(a)

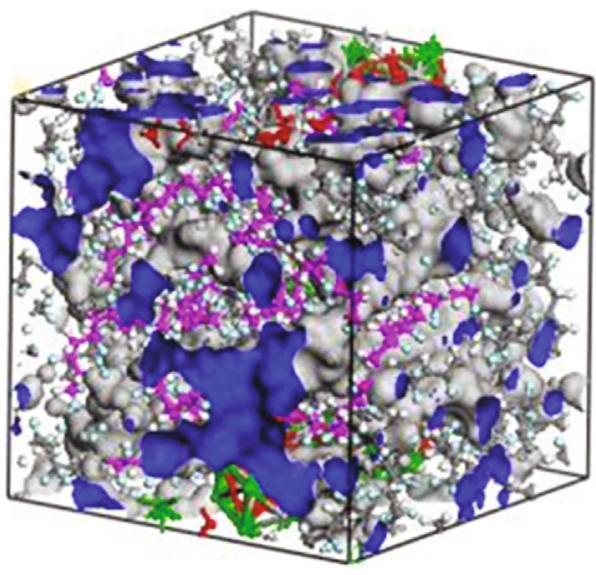

(c)

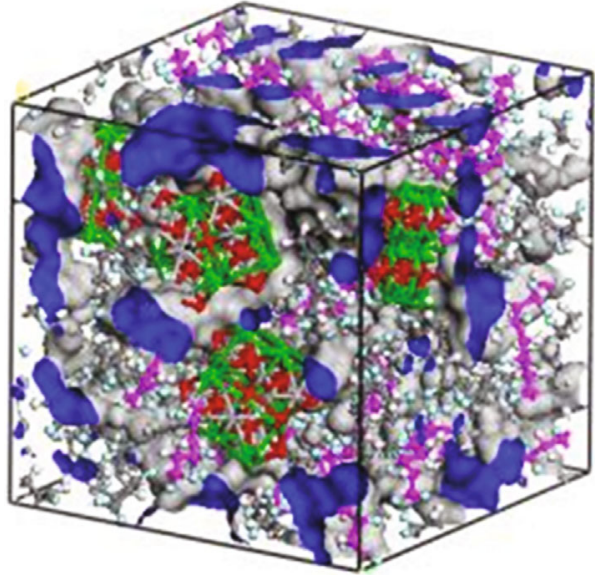

(b)

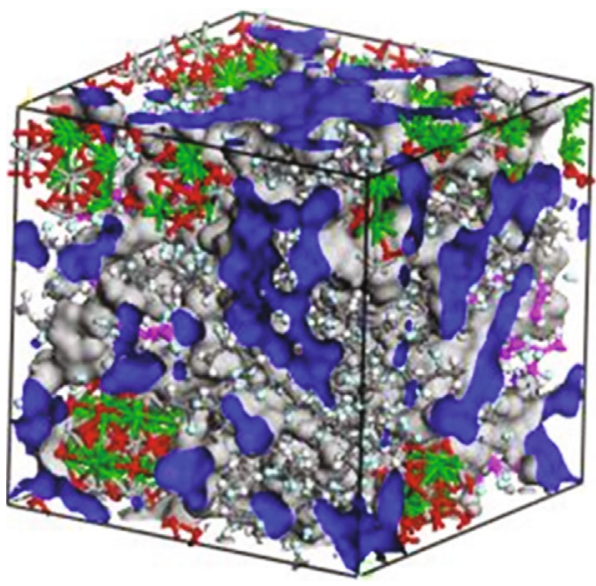

(d)

Figure 8: The spatial structure of PVDF (a), BT10/PVDF (b), BT15/PVDF (c), and BT20/PVDF (d) by MD simulation. The free volume is shown in blue, and the occupied volume in gray.

TABLE 3: Free volume fraction.

\begin{tabular}{lcccc}
\hline Sample & $V_{\text {free }}\left(\AA^{3}\right)$ & $V_{\text {occupied }}\left(\AA^{3}\right)$ & $V_{\text {total }}\left(\AA^{3}\right)$ & FFV $(\%)$ \\
\hline PVDF & 6511.31 & 16562.49 & 23073.80 & $28.22 \%$ \\
BT10/PVDF & 5543.51 & 21305.25 & 26848.76 & $20.65 \%$ \\
BT15/PVDF & 6239.67 & 21931.98 & 28171.65 & $22.15 \%$ \\
BT20/PVDF & 7477.57 & 22487.03 & 29964.60 & $24.95 \%$ \\
\hline
\end{tabular}

was cooled down to $150 \mathrm{~K}$ at a temperature interval of $40 \mathrm{~K}$. Secondly, 100 ps NVT and 300 ps NPT simulations at each temperature were performed, and then, the final $150 \mathrm{ps}$ NPT dynamic trajectory was taken to calculate the specific volume. The curves of the specific volume vs. temperature are shown in Figure 9.

As seen in Figure 9, $T_{\mathrm{g}}$ of BT10/PVDF is $80.71 \mathrm{~K}$ higher than that of PVDF, and $T_{\mathrm{g}}$ of the composites tends to decrease with the increasing BT content from $10 \mathrm{vol} \%$ to $20 \mathrm{vol} \%$. When the BT content is $20 \mathrm{vol} \%, T_{\mathrm{g}}$ of BT20/PVDF is $47.08 \mathrm{~K}$ lower than that of BT10/PVDF; however, it is still $33.63 \mathrm{~K}$ higher than that of PVDF. These results show good consistence with those of FFV, which can be well explained according to the glass transition theory [36]. The higher $T_{\mathrm{g}}$ could be a result of the decreased FFV, which signifies the confined segment motion. For BT10/PVDF, BT particles (shown in Figures 5(d) and 6(a)) occupy the free volume (shown in Figure 8(b)) and decrease the FFV (shown in Table 3), which hinders the segment motion and gives rise to the higher $T_{\mathrm{g}}$ than PVDF. As the BT content increases to $20 \mathrm{vol} \%$, the agglomeration of BT particles (shown in Figures 5(f) and 6(c)) results in the increase of the free volume (shown in Figure $8(\mathrm{~d})$ ) and the FFV (shown in Table 3), which provides more space for the segment motion and gives rise to the lower $T_{\mathrm{g}}$ than BT10/PVDF. Moreover, the BT particles increase the density of entanglement points of the macromolecular chains and restrict the segment motion, as a result of which $T_{\mathrm{g}}$ rises. Thus, the higher $T_{\mathrm{g}}$ of BT20/PVDF than PVDF can be contributed to the lower FFV than PVDF and the restriction effect of the BT particles on the segment motion. The similar results were reported by Nerantzaki et al. in their investigation of polylactic acid/silica nanocomposites [37].

In view of the simulation results of the FFV and $T_{\mathrm{g}}$ and the morphological structure of the MD simulations and the 


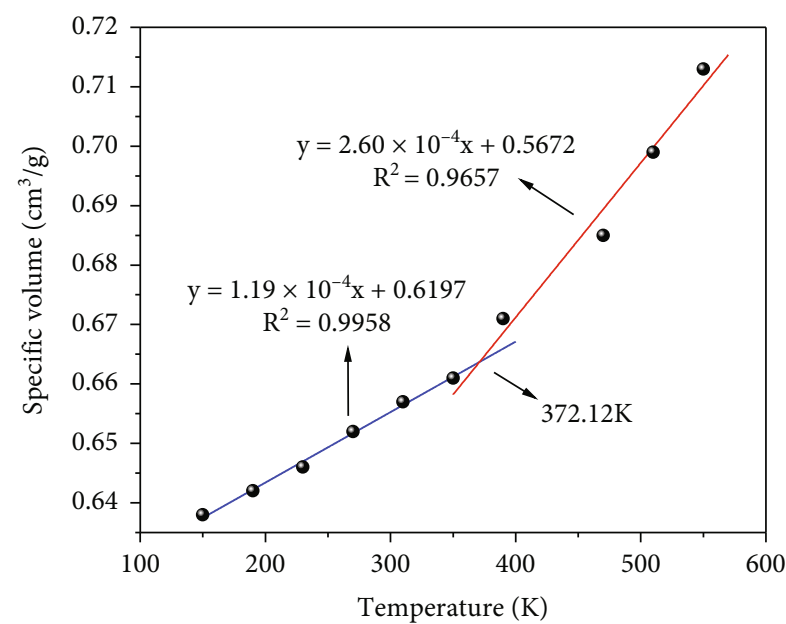

- PVDF

(a)

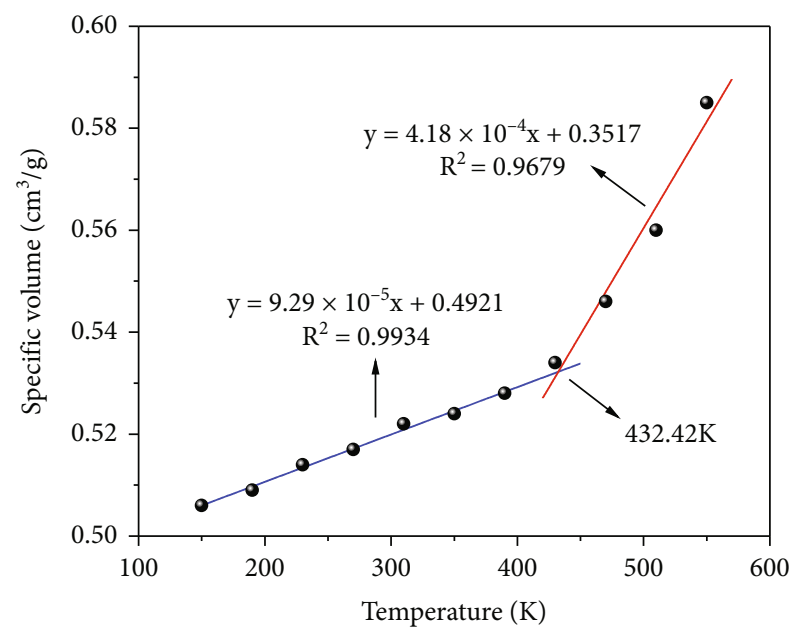

- BT15/PVDF

(c)

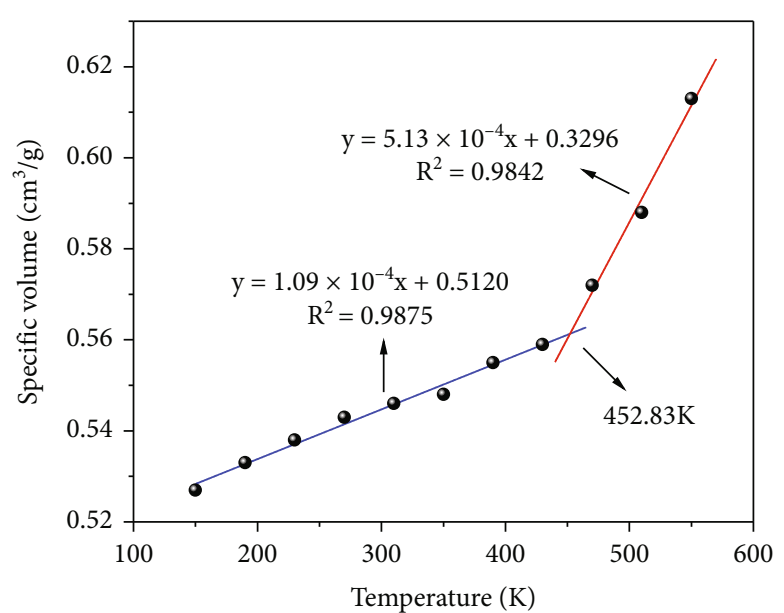

- BT10/PVDF

(b)

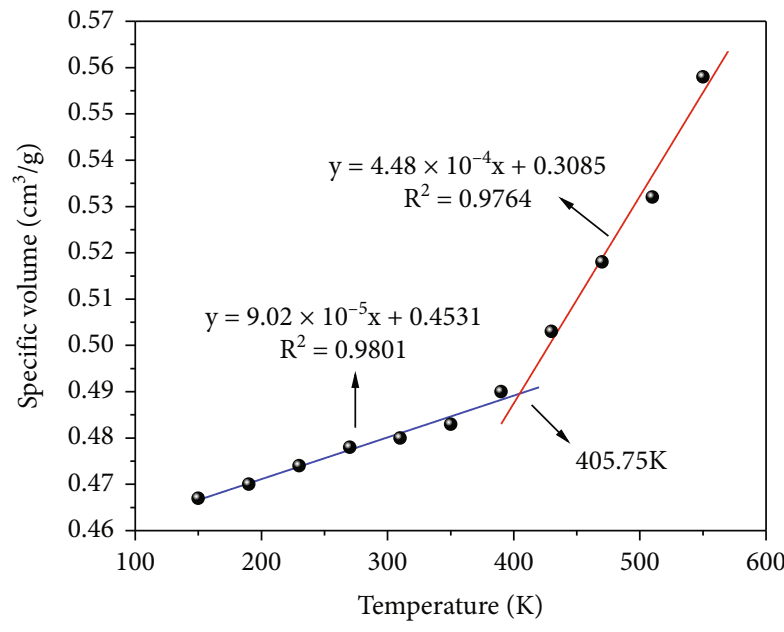

- BT20/PVDF

Figure 9: The specific volume vs. temperature curves of PVDF (a), BT10/PVDF (b), BT15/PVDF (c), and BT20/PVDF (d) by MD simulation.

SEM observations, the high consistency between the experimental results and the simulations is revealed, which provides the evidences for the rationality of the model and the accuracy of the calculation. On the basis of the MD simulations, the dielectric permittivity of the composites was studied.

\subsection{Dielectric Permittivity}

4.3.1. Dielectric Spectroscopy. Dielectric spectroscopy is a powerful tool for the characterization of dielectric materials by the measurement of the permittivity as a function of frequency and temperature. Figure 10 shows the curves of the real part of the complex permittivity $\left(\varepsilon^{\prime}\right)$ and the loss factor $(\tan \delta)$ of PVDF and BT/PVDF composites as a function of frequency. As shown in Figure 10(a), the curves of $\varepsilon^{\prime}$ show a stepped downward trend with the increase of the frequency. A rapid decrease of $\varepsilon^{\prime}$ was found at $1-10^{2} \mathrm{~Hz}$, which is attributed to the effect of interface polarization. As the frequency increases from $10^{2} \mathrm{~Hz}$ to $10^{5} \mathrm{~Hz}$, only a slight decrease of $\varepsilon^{\prime}$ is found. A significant decrease of $\varepsilon^{\prime}$ was found at $10^{5}-10^{7} \mathrm{~Hz}$, which is attributed to the effect of dipole orientation polarization. The response of the dielectric constant of the BT/PVDF composite to frequency is mainly due to the influence of different polarization mechanisms. With the introduction of BT particles, $\varepsilon^{\prime}$ of BT/PVDF composites shows marked increase with the BT contents. At $1 \mathrm{kHz}, \varepsilon^{\prime}$ is 8.98 for PVDF, 13.91 for BT10/PVDF, 17.76 for BT15/PVDF, and 19.38 for BT20/PVDF, respectively. An increase of $\varepsilon^{\prime}$ about $115 \%$ can be found while comparing between BT20/PVDF and PVDF. When the BT content increases from $10 \mathrm{vol} \%$ to $20 \mathrm{vol} \%, \varepsilon^{\prime}$ increases by about $40 \%$ and the increasement rate of $\varepsilon^{\prime}$ slows down, which can be explained by the agglomeration of BT particles. 


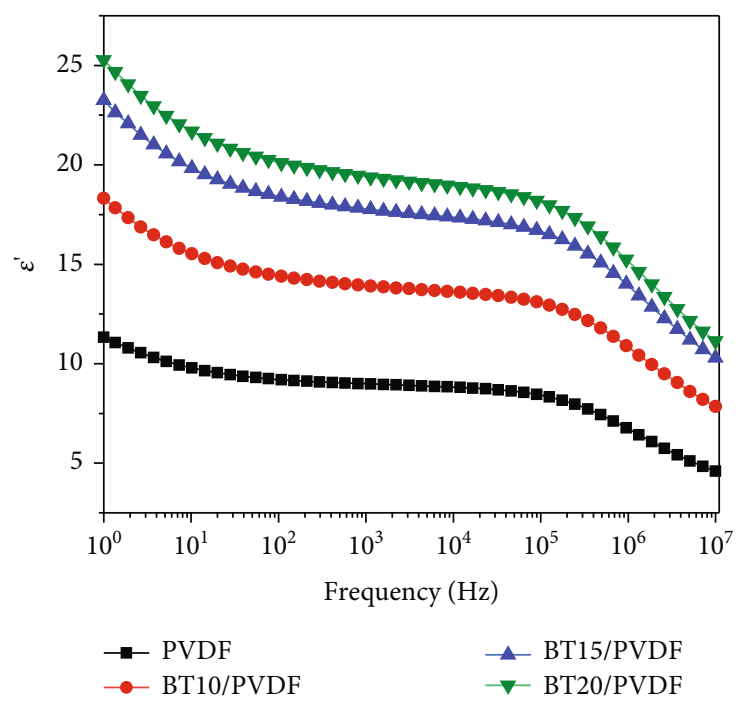

(a)

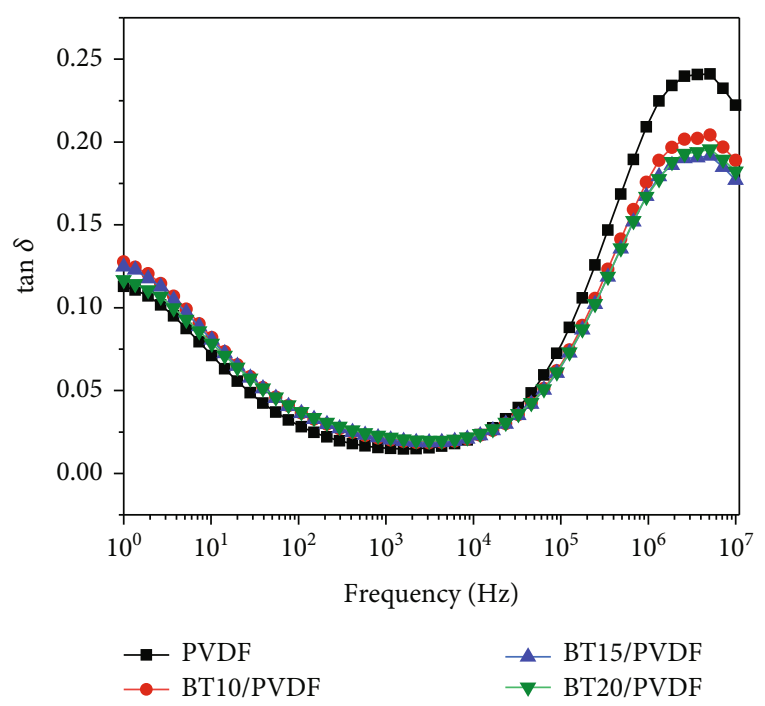

(b)

Figure 10: The real part of the complex permittivity (a) and the loss factor (b) of PVDF and BT/PVDF composites as a function of frequency.

Two peaks of $\tan \delta$ corresponding to the interface polarization and the dipole orientation polarization are found at the frequency range of $1-10^{2} \mathrm{~Hz}$ and $10^{5}-10^{7} \mathrm{~Hz}$ in Figure 10(b), respectively. The composites with different BT contents show the similar $\tan \delta$ curves as a function of frequency. The same frequency corresponding to the peak of $\tan \delta$ due to dipole orientation polarization for PVDF and BT/PVDF composites is found, indicating the same polarization mechanism. At $1 \mathrm{~Hz}, \tan \delta$ of the composites is slightly higher than that of PVDF, which can be explained by the enhanced interface polarization due to the addition of BT particles. At $1 \mathrm{kHz}, \tan \delta$ of the composites tends to be the same as that of PVDF due to the similar polarization process. At $1 \mathrm{MHz}, \tan \delta$ of the composites is lower than that of PVDF, which can be explained by the impaired dipole orientation polarization due to the decreased content of PVDF.

\subsubsection{Permittivity by Cole-Cole Equation. Figure 11 shows} the relationship between the real part $\left(\varepsilon^{\prime}\right)$ and the imaginary part $\left(\varepsilon^{\prime \prime}\right)$ of the complex permittivity for PVDF and BT/PVDF composites. It can be seen that the curves consist of two parts, a circular arc (the left part of the curve) and a straight-like line (the right part of the curve). The right part is related to the interface polarization at low frequencies, while the left part is related to the dipole orientation polarization at high frequencies. The circular arc at the left reveals $\varepsilon^{\prime \prime}$ decreases as $\varepsilon^{\prime}$ increases, which corresponds to the increase of $\varepsilon^{\prime \prime}$ at high frequency. In this scenario, $\varepsilon^{\prime}-\varepsilon^{\prime \prime}$ curve is mainly dependent on the dipole orientation polarization, which reflects the dielectric relaxation of the composites. Dielectric spectroscopy is sensitive to dipolar relaxations, which are usually analyzed using theoretical models based on the well-founded Debye equation to describe the experimental spectroscopy. Hereinaf- ter, the Cole-Cole equation as shown in Equation (2) was adopted to describe the dielectric relaxation of BT/PVDF composites according to the method reported by Wang et al. in the study of carbon nanotube/epoxy composites [38].

$$
\varepsilon^{*}=\varepsilon^{\prime}-j \varepsilon^{\prime \prime}=\varepsilon_{\infty}+\frac{\varepsilon_{\mathrm{s}}-\varepsilon_{\infty}}{1+j \omega \tau^{(1-\alpha)}},
$$

where $\varepsilon^{*}$ is the complex permittivity, $\varepsilon_{s}$ is the static permittivity, $\varepsilon_{\infty}$ is the optical frequency permittivity, $\tau$ is the average relaxation time, and $\alpha(0 \leq \alpha \leq 1)$ represents the dispersion of the relaxation time.

The Cole-Cole equation is used to fit the circular arc in the $\varepsilon^{\prime}-\varepsilon^{\prime \prime}$ curves, and the fitted curves are shown in the solid line in Figure 11. The calculated values of the relevant parameters are shown in Table 4 . It can be seen that $\varepsilon_{\mathrm{s}}$ and $\varepsilon_{\infty}$ increase with the increasing BT content while $\tau$ and $\alpha$ show no significant changes. Accordingly, there are no or few effects of BT particles on the dielectric relaxation of PVDF at high frequency, which is originated from the dipole orientation polarization of PVDF. The $\mathrm{BT} / \mathrm{PVDF}$ composites present the same dielectric relaxation process as PVDF, and the increased permittivity of the composites is mainly due to the high permittivity of the BT particles.

4.3.3. Permittivity by MD Simulation. Based on the principle of statistical mechanics and the fluctuation dissipation theorem, a script was written to calculate the total dipole moment of the model system. The MD simulation mainly considers the influence of polarization on the dielectric properties. According to the contribution of the dipole orientation polarization to the permittivity, the theoretical static permittivity is calculated by Equation (3) [39]. The result of Equation (3) is usually 


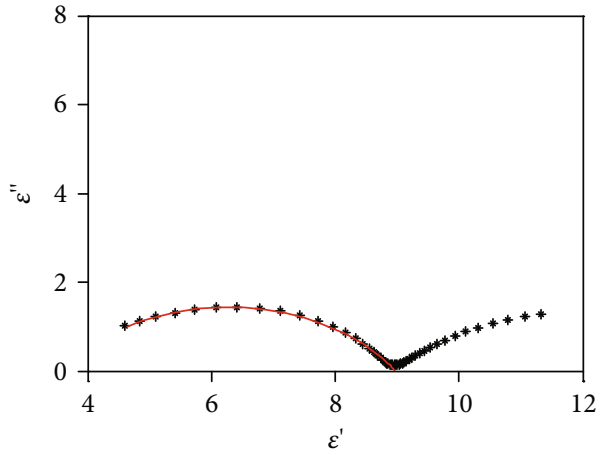

(a)

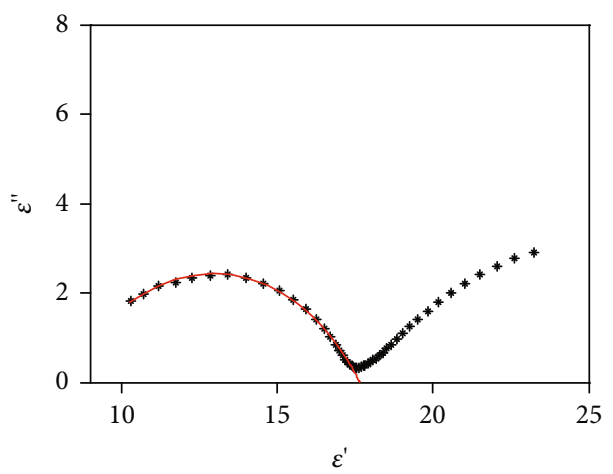

(c)

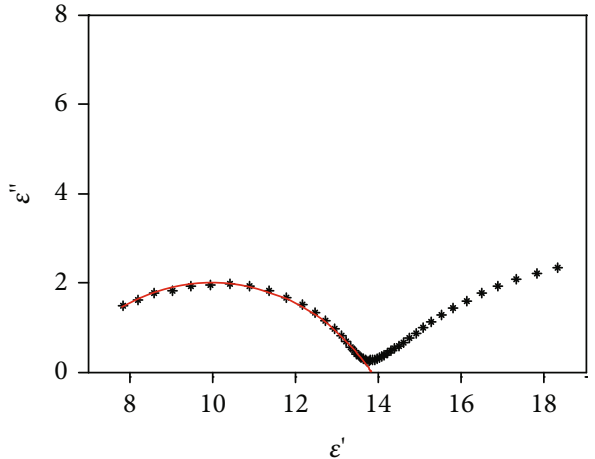

(b)

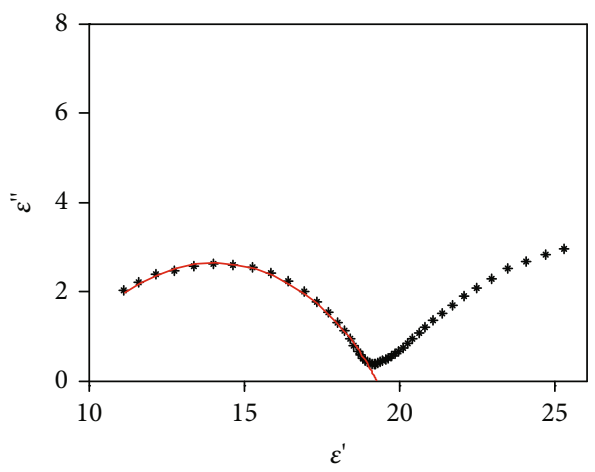

(d)

Figure 11: Cole-Cole curves of PVDF (a), BT10/PVDF (b), BT15/PVDF (c), and BT20/PVDF (d). The line represents the fitted curve by the Cole-Cole equation.

TABle 4: The calculated parameters by the Cole-Cole equation.

\begin{tabular}{lcccc}
\hline Sample & $\varepsilon_{\mathrm{s}}$ & $\varepsilon_{\infty}$ & $\tau(\mathrm{s})$ & $\alpha$ \\
\hline PVDF & 8.97 & 3.58 & $0.96 \times 10^{-7}$ & 0.374 \\
BT10/PVDF & 13.83 & 6.15 & $0.86 \times 10^{-7}$ & 0.390 \\
BT15/PVDF & 17.65 & 8.23 & $0.86 \times 10^{-7}$ & 0.393 \\
BT20/PVDF & 19.26 & 8.80 & $0.84 \times 10^{-7}$ & 0.405 \\
\hline
\end{tabular}

corrected by Equation (4) [40].

$$
\varepsilon_{\mathrm{MD}}=1+\frac{\left.<M^{2}>-<M\right\rangle^{2}}{3 \varepsilon_{0} V k_{\mathrm{B}} T},
$$

where $\varepsilon_{\mathrm{MD}}$ is the theoretical static permittivity; $\langle M>$ is the total dipole moment; $\varepsilon_{0}$ is the vacuum permittivity; $V$ is the volume of the system; $k_{\mathrm{B}}$ is the Boltzmann constant; and $T$ is the temperature.

$$
\varepsilon_{\mathrm{MDV}}=\varepsilon_{\mathrm{v}}+\frac{<M^{2}>-<M>^{2}}{3 \varepsilon_{0} V k_{\mathrm{B}} T}
$$

where $\varepsilon_{\mathrm{MDV}}$ is the modified theoretical static permittivity and $\varepsilon_{\mathrm{v}}$ is calculated by [40]

$$
\frac{\varepsilon_{\mathrm{v}}-1}{\varepsilon_{\mathrm{v}}+2}=\frac{4 \pi \chi}{3<V>},
$$

where $\langle V\rangle$ is the average molecular volume and $\chi$ is the polarizability.

Considering the relationship between the polarization intensity $(P)$ and the external electric field $(E)$ and $\chi$, shown in Equation (6) and Equation (7), the external electric field can be used to calculate $\varepsilon_{\mathrm{v}}$, and then $\varepsilon_{\mathrm{MDV}}$ can be obtained. To simplify the correction process, $\langle V\rangle$ was determined according to Equation (8).

$$
\begin{gathered}
P=\frac{<M>}{V}, \\
P=\chi E, \\
<V>=\frac{V \varphi}{N_{1}}+\frac{V(1-\varphi)}{N_{2}},
\end{gathered}
$$

where $\varphi$ is the volume fraction of BT; $N_{1}$ is the number of BT particles in the model; and $N_{2}$ is the number of PVDF molecular chains in the model.

The external electric field was calculated according to Equation (9) with the voltage applied during the characterization of dielectric spectroscopy. In order to simplify the correction process of the static permittivity by MD simulation, no other corrections were adopted to modify the effective electric field.

$$
E=\frac{U}{d}
$$


TABLE 5: Calculated parameters for MD simulations of BT/PVDF composites.

\begin{tabular}{|c|c|c|c|c|c|}
\hline Sample & $V\left(\AA^{3}\right)$ & $<V>\left(\AA^{3}\right)$ & $\chi\left(\mathrm{cm}^{3}\right)$ & $E(\mathrm{~V} / \mathrm{mm})$ & $d(\mathrm{~mm})$ \\
\hline BT10/PVDF & $3.88 \times 10^{4}$ & 12933.3 & $1.53 \times 10^{-21}$ & 9.68 & 0.31 \\
\hline BT15/PVDF & $4.06 \times 10^{4}$ & 13025.8 & $1.79 \times 10^{-21}$ & 10.00 & 0.30 \\
\hline BT20/PVDF & $4.13 \times 10^{4}$ & 12390.0 & $1.80 \times 10^{-21}$ & 11.54 & 0.26 \\
\hline
\end{tabular}

TABLE 6: Static permittivity of BT/PVDF composites obtained from Equation (2) $\left(\varepsilon_{\mathrm{s}}\right)$, Equation (3) $\left(\varepsilon_{\mathrm{MD}}\right)$, and Equation (4) $\left(\varepsilon_{\mathrm{MDV}}\right)$.

\begin{tabular}{lccc}
\hline Sample & $\varepsilon_{s}$ & $\varepsilon_{\text {MD }}$ & $\varepsilon_{\text {MDV }}$ \\
\hline BT10/PVDF & 13.83 & 10.76 & 13.64 \\
BT15/PVDF & 17.65 & 13.18 & 17.32 \\
BT20/PVDF & 19.26 & 15.52 & 20.02 \\
\hline
\end{tabular}

where $U$ is the voltage during the test of dielectric spectroscopy and $d$ is the thickness of the sample.

BT is a typical ferroelectric material with spontaneous polarization, which mainly originates from ion displacement polarization and electron displacement polarization. In the absence of an external electric field, the spontaneous polarization directions are randomly distributed and no dipole moments are observed. Under an external electric field, the spontaneous polarization can be adjusted along the direction of the electric field to form dipole moments. For BT/PVDF composites, the dipole moments generated by BT particles would effectively increase the permittivity of the composites. To perform the MD simulation, $\langle M>$ was continued to drop from a relatively large value (about $200 \mathrm{D}$ ) to 5-7 D, approaching the ideal limit for "zero net dipole moment." During the correction process, the testing value of the external electric field was introduced to make a comparison between the results of the MD simulations and the dielectric spectroscopy. Table 5 shows the calculated parameters by MD simulation, and Table 6 shows the static permittivity calculated by $\mathrm{MD}$ simulation together with that by Cole-Cole equation.

In Table $6, \varepsilon_{\mathrm{MD}}$ is the static permittivity obtained by MD simulation, $\varepsilon_{\mathrm{MDV}}$ is the static permittivity corrected by the testing electric field, and $\varepsilon_{\mathrm{s}}$ is the static permittivity obtained by fitting the $\varepsilon^{\prime}-\varepsilon^{\prime \prime}$ curves according to the ColeCole equation. It can be seen that both $\varepsilon_{\mathrm{MD}}$ and $\varepsilon_{\mathrm{MDV}}$ increase with the increase of BT content, which coincides with the results of dielectric spectroscopy. Moreover, $\varepsilon_{\mathrm{MDV}}$ is very close to $\varepsilon_{\mathrm{s}}$ in value, indicating the very good consistency among the static permittivity corrected by the applied electric field and that obtained by the Cole-Cole fitting of the dielectric spectroscopy. As a result, the permittivity of BT/PVDF composites can be successfully predicted by the simulation model and the calculation method. The proposed MD simulation acts as a bridge between the microscopic polarization mechanism and the macroscopic dielectric properties and would be in favor of the investigation and the design of dielectric composites.

\section{Conclusions}

In this work, we reported the MD simulations on the structure, glass transition temperature, and dielectric permittivity of BT/PVDF composites. The initial models which are composed of three PVDF macromolecular chains and BT particles were proposed on the basis of the crystalline structure of PVDF, BT, and BT/PVDF composites. The models were proved to be reasonable and practical in the $\mathrm{MD}$ simulations on the structure of BT/PVDF composites. As a result, the simulated structure showed well consistence with the morphological observation by SEM and revealed the assembling of PVDF macromolecular chains and the agglomeration of BT particle with the increasing content of BT in the composites. Thanks to this featured structure of the composites, the segment motion and the conformation of the macromolecular chain were influenced, which were affirmed by the MD simulations on FFV and $T_{\mathrm{g}}$. The composites presented the higher $T_{\mathrm{g}}$ than PVDF due to the lower FFV and the restriction effect of the BT particles on the segment motion. Meanwhile, the physical interaction between BT and PVDF was suggested according to the simulated structure and the experimental results of XRD and SEM. More evidences came from the same frequency corresponding to the peak of $\tan \delta$ due to dipole orientation polarization and the average relaxation time $(\tau)$ of the same order by Cole-Cole fitting while investigating the dielectric spectroscopy. Furthermore, the static dielectric permittivity was calculated by the molecular dynamics simulation statistical dipole moment and corrected by the electric field during the testing of dielectric spectroscopy. The calculated results were found in good agreement with the static dielectric permittivity obtained by the Cole-Cole fitting of the dielectric spectroscopy. Accordingly, the rationality of the model and the accuracy of the calculation were indicated. The structure and the dielectric permittivity of BT/PVDF composites can be successfully simulated by the proposed simulations and the calculation models. MD simulations would be helpful in the research of dielectric composites by acting as a bridge between the micropolarization mechanism and the macrodielectric properties.

\section{Data Availability}

The data used to support the findings of this study are included within the article.

\section{Conflicts of Interest}

There are no conflicts to declare. 


\section{Acknowledgments}

The authors acknowledge supports from the Key projects of Natural Science Foundation of Heilongjiang Province (ZD2020E007).

\section{Supplementary Materials}

Supplementary 1. Supplementary figure 1: the temperature and energy distribution during the final NPT-MD simulation of BT10/PVDF and BT15/PVDF hybrid systems. It is proved that the MD simulation of BT10/PVDF and BT15/PVDF models reaches equilibrium.

Supplementary 2. The original data file of material dielectric properties is the original data of $\varepsilon^{\prime}, \varepsilon^{\prime \prime}$, and $\tan \delta$ of PVDF, BT10/PVDF, BT15/PVDF, and BT20/PVDF composites.

Supplementary 3. The original data file of the glass transition temperature is the original data of the parameters related to the glass transition temperature of PVDF, BT10/PVDF, BT15/PVDF, and BT20/PVDF composite materials calculated by MD simulation.

\section{References}

[1] Prateek, V. K. Thakur, and R. K. Gupta, "Recent Progress on Ferroelectric Polymer-Based Nanocomposites for High Energy Density Capacitors: Synthesis, Dielectric Properties, and Future Aspects," Chemical Reviews, vol. 116, no. 7, pp. 42604317, 2016.

[2] L. Wang, H. Luo, X. Zhou et al., "Enhanced permittivity and energy density of $\mathrm{P}(\mathrm{VDF}-\mathrm{HFP})$-based capacitor using coreshell structured BaTiO3@TiO2 fillers," Ionics, vol. 24, no. 12, pp. 3975-3982, 2018.

[3] X. Zhang, Y. Ma, C. Zhao, and W. Yang, "High dielectric constant and low dielectric loss hybrid nanocomposites fabricated with ferroelectric polymer matrix and $\mathrm{BaTiO} 3$ nanofibers modified with perfluoroalkylsilane," Applied Surface Science, vol. 305, no. 30, pp. 531-538, 2014.

[4] Y. Wang, X. Wu, C. Feng, and Q. Zeng, "Improved dielectric properties of surface modified $\mathrm{BaTiO} 3 /$ polyimide composite films," Microelectronic Engineering, vol. 154, no. 25, pp. 1721, 2016.

[5] Z. Yao, Z. Song, H. Hao et al., "Homogeneous/InhomogeneousStructured Dielectrics and their Energy-Storage Performances," Advanced Materials, vol. 29, no. 20, article 1601727, 2017.

[6] K. Bi, M. Bi, Y. Hao et al., "Ultrafine core-shell BaTiO3@SiO2 structures for nanocomposite capacitors with high energy density," Nano Energy, vol. 51, pp. 513-523, 2018.

[7] K. Zou, Y. Dan, H. Xu et al., "Recent advances in lead-free dielectric materials for energy storage," Materials Research Bulletin, vol. 113, pp. 190-201, 2019.

[8] Z. Dang, J. Yuan, J. Zha, P. Hu, D. Wang, and Z. Cheng, "Highpermittivity polymer nanocomposites: Influence of interface on dielectric properties," Journal of Advanced Dielectrics, vol. 3, pp. 1-8, 2013.

[9] X. Lin, P. Hu, Z. Jia, and S. Gao, "Enhanced electric displacement induces large energy density in polymer nanocomposites containing core-shell structured BaTiO3@TiO2nanofibers," Journal of Materials Chemistry A, vol. 4, no. 6, pp. 23142320, 2016.
[10] P. Hu, S. Gao, Y. Zhang, L. Zhang, and C. Wang, "Surface modified $\mathrm{BaTiO}_{3}$ nanoparticles by titanate coupling agent induce significantly enhanced breakdown strength and larger energy density in PVDF nanocomposite," Composites Science and Technology, vol. 156, no. 1, pp. 109-116, 2018.

[11] Z. Zhang, Y. Gu, S. Wang, M. Li, J. Bi, and Z. Zhang, "Enhancement of dielectric and electrical properties in $\mathrm{BT} / \mathrm{SiC} / \mathrm{PVDF}$ three-phase composite through microstructure tailoring," Composites Part A: Applied Science and Manufacturing, vol. 74, pp. 88-95, 2015.

[12] Y. Li, Y. Shi, F. Cai, J. Xue, F. Chen, and Q. Fu, "Graphene sheets segregated by barium titanate for polyvinylidene fluoride composites with high dielectric constant and ultralow loss tangent," Composites Part A: Applied Science and Manufacturing, vol. 78, pp. 318-326, 2015.

[13] Y. Yang, C. Sun, H. Deng, and Q. Fu, "Ni(OH)2 as an novel shell layer material for core-shell dielectric filler based on barium titanate and their dielectric polymer composites in P(VDF-HFP) matrix," Composites Science and Technology, vol. 198, no. 29, p. 108274, 2020.

[14] D. Zhang, Z. Wu, X.-f. Zhou, A.-q. Wei, C. Chen, and H. Luo, "High energy density in $\mathrm{P}(\mathrm{VDF}-\mathrm{HFP})$ nanocomposite with paraffin engineered $\mathrm{BaTiO} 3$ nanoparticles," Sensors and Actuators A: Physical, vol. 260, no. 15, pp. 228-235, 2017.

[15] Y. Jiang, Z. Zhang, Z. Zhou, H. Yang, and Q. Zhang, "Enhanced Dielectric Performance of P(VDF-HFP) Composites with Satellite-Core-Structured Fe2O3@BaTiO3 Nanofillers," Polymers, vol. 11, no. 10, p. 1541, 2019.

[16] S. Ishaq, F. Kanwal, S. Atiq et al., "Dielectric and impedance spectroscopic studies of three phase graphene/titania/poly(vinyl alcohol) nanocomposite films," Results in Physics, vol. 11, pp. 540-548, 2018.

[17] H. Jia, W. Zhou, H. Nan et al., "Enhanced high temperature dielectric polarization of barium titanate/magnesium aluminum spinel composites and their potential in microwave absorption," Journal of the European Ceramic Society, vol. 40, no. 3, pp. 728-734, 2020.

[18] A. Mahmood, A. Naeem, and T. Mahmood, Properties and Applications of Polymer Dielectrics, Intech Open, 2017.

[19] J. Ma, U. Azhar, C. Zong et al., "Core-shell structured PVDF@BT nanoparticles for dielectric materials: A novel composite to prove the dependence of dielectric properties on ferroelectric shell," Materials \& Design, vol. 164, article 107556, 2019.

[20] Z. Wang, T. Wang, C. Wang, and Y. J. Xiao, "Mechanism of enhanced dielectric performance in $\mathrm{Ba}(\mathrm{Fe} 0.5 \mathrm{Ta} 0.5) \mathrm{O} 3$ /poly(vinylidene fluoride) nanocomposites," Ceramics International, vol. 43, no. 1, pp. S244-S248, 2017.

[21] G. Hu, F. Gao, J. Kong et al., "Preparation and dielectric properties of poly(vinylidene fluoride)/Ba0.6Sr0.4TiO3 composites," Journal of Alloys and Compounds, vol. 619, pp. 686692, 2015.

[22] Y. Thakur, R. Dong, M. Lin et al., "Optimizing nanostructure to achieve high dielectric response with low loss in strongly dipolar polymers," Nano Energy, vol. 16, pp. 227-234, 2015.

[23] R. P. Magisetty, A. Shukla, and B. Kandasubramanian, "Molecular Dynamic Simulation Constructed Interaction Parameter Investigation between Poly(1,6-heptadiyne) and $\mathrm{NiFe} 2 \mathrm{O} 4$ in Nanocomposite," Materials Today: Proceedings, vol. 24, no. 3, pp. 1720-1728, 2020. 
[24] C. Wang, G. Pilania, S. A. Boggs, S. Kumar, C. Breneman, and R. Ramprasad, "Computational strategies for polymer dielectrics design," Polymer, vol. 55, no. 4, pp. 979-988, 2014.

[25] Y. Feng, H. Zou, M. Tian, L. Zhang, and J. Mi, "Relationship between Dispersion and Conductivity of Polymer Nanocomposites: A Molecular Dynamics Study," Physical chemistry B, vol. 116, no. 43, pp. 13081-13088, 2012.

[26] X. Liu, K. Cheng, and G. Jia, "Investigation of nonlinear dielectric response of DMSO-methanol mixture by molecular dynamics simulation," Journal of Molecular Liquids, vol. 294, no. 15, article 111678, 2019.

[27] F. Liu, W. Tian, X. Yang, and G. Z. Jia, "Hydrogen-bonding and dielectric response of $\mathrm{N}, \mathrm{N}$-dimethylacetamide aqueous solutions under E/M fields using molecular dynamics," Journal of Molecular Liquids, vol. 197, pp. 100-105, 2014.

[28] L. Shen, L. Zou, M. Ding, T. Zhao, L. Zhang, and Q. Li, "Investigation of physical properties of epoxy-functionalized graphene nanoplatelets composite coatings on DC-GIL insulators by molecular dynamics simulation," Applied Surface Science, vol. 505, article 144197, 2020.

[29] Y. Huang, C. Chen, Y. Tseng et al., "Graphene Wrinkles affect electronic transport in nanocomposites: Insight from molecular dynamics simulations," Journal of Molecular Graphics and Modelling, vol. 92, pp. 236-242, 2019.

[30] Y. Feng, P. Liang, B. Tang et al., "Construction of particle network for ultrahigh permittivity of dielectric polymer composite toward energy devices: A molecular dynamics study," Nano Energy, vol. 64, article 103985, 2019.

[31] R. Senthil Kumar, T. Sarathi, K. K. Venkataraman, and A. Bhattacharyya, "Enhanced piezoelectric properties of polyvinylidene fluoride nanofibers using carbon nanofiber and electrical poling," Materials Letters, vol. 255, article 126515, 2019.

[32] A. Thakur, S. Dam, and S. Hussain, "Probing the effect of CdS loading on crystallinity and morphology of free standing thin films of CdS/PVDF," Materials Today: Proceedings, vol. 46, pp. 6156-6160, 2021.

[33] H. S. Mohanty, A. K. Ravikant, A. Kumar, P. K. Kulriya, R. Thomas, and D. K. Pradhan, "Dielectric/ferroelectric properties of ferroelectric ceramic dispersed poly(vinylidene fluoride) with enhanced $\beta$-phase formation," Materials Chemistry and Physics, vol. 230, pp. 221-230, 2019.

[34] A. Bottino, G. Capannelli, S. Munari, and A. Turturro, "Solubility parameters of poly(vinylidene fluoride)," Journal of Polymer Science Part A Polymer Chemistry, vol. 26, no. 4, pp. 785794, 1988.

[35] B. Chen, Y. Dai, X. Ruan, Y. Xi, and G. He, "Integration of molecular dynamic simulation and free volume theory for modeling membrane VOC/gas separation," Frontiers of Chemical Science and Engineering, vol. 12, no. 2, pp. 296-305, 2018.

[36] X. Wang, X. Zhao, J.-W. He, T. W. Chan, X.-Z. Cao, and S.$\mathrm{Z}$. Wu, "Thermodynamic Properties and Free Volume Analyses of Polycarbonates by a Combined Experimental and Molecular Simulation Method," Industrial \& Engineering Chemistry Research, vol. 54, no. 25, pp. 6578-6588, 2015.

[37] M. Nerantzaki, L. Prokopiou, D. N. Bikiaris et al., "In situ prepared poly(DL-lactic acid)/silica nanocomposites: Study of molecular composition, thermal stability, glass transition and molecular dynamics," Thermochimica Acta, vol. 669, no. 10, pp. 16-29, 2018.
[38] B. Wang, L. Liu, L. Huang et al., "Fabrication and origin of high-k carbon nanotube/epoxy composites with low dielectric loss through layer-by-layer casting technique," Carbon, vol. 85, pp. 28-37, 2015.

[39] M. Neumann, "Dipole moment fluctuation formulas in computer simulations of polar systems," Molecular Physics, vol. 50, no. 4, pp. 841-858, 1983.

[40] O. Gereben and L. Pusztai, "On the accurate calculation of the dielectric constant from molecular dynamics simulations: The case of SPC/E and SWM4-DP water," Chemical Physics Letters, vol. 507, no. 1-3, pp. 80-83, 2011. 